\title{
Ionization of highly charged relativistic ions by neutral atoms and ions
}

\author{
G. Baur \\ Institut für Kernphysik and Jülich Centre for Hadron Physics, Forschungszentrum Jülich, D-52425 Jülich, Germany
}

I. L. Beigman, V. P. Shevelko, and I. Yu. Tolstikhina

P. N. Lebedev Physical Institute, Leninskii Prospekt 53, 119991 Moscow, Russia

Th. Stöhlker

Gesellschaft für Schwerionenforschung (GSI) mbH, Planckstrasse 1, D-64291 Darmstadt, Germany

(Received 10 November 2008; revised manuscript received 4 May 2009; published 24 July 2009)

\begin{abstract}
Ionization of highly charged relativistic ions by neutral atoms and ions is considered. Numerical results of recently developed computer codes based on the plane-wave Born and the equivalent-photon approximations are presented and compared with experimental data available at relativistic energies for $1 \mathrm{~s}$ electron ionization. New formulas for relativistic cross sections are derived for ionization of the projectile electron with arbitrary quantum numbers $n$ and $l$. The results are obtained on the basis of the nonrelativistic reduction in the Dirac matrix element.
\end{abstract}

DOI: 10.1103/PhysRevA.80.012713

PACS number(s): $34.10 .+\mathrm{x}, 34.50 .-\mathrm{s}$

\section{INTRODUCTION}

Projectile ionization, also referred to as electron loss or stripping, is an important charge-changing process playing a critical role in many applications such as heavy-ion driven inertial fusion, ion-beam lifetimes in accelerators, medical science, material technology, and others (see, e.g., [1-3]).

Relativistic ionization has been pioneered at Stanford by Anholt [4] and it is reviewed in several books and review articles (see, e.g., [5-7]). In the literature, experimental data and numerical calculations of relativistic ionization cross sections are presented mainly for ionization of $\mathrm{H}-$ and $\mathrm{He}-$ like ions from the ground $1 s$ state (see, e.g., [8-17]).

Recently, two new computer codes Loss-R (for relativistic loss) and HERION (for high-energy relativistic ionization) have been developed for calculation of the relativistic ionization cross sections for arbitrary $n l$ states of the projectile. The LOSS-R code [18] was created on the basis of the nonrelativistic LOSS code [19] using the plane-wave Born approximation (PWBA) in the momentum-transfer representation and the Schrödinger wave functions while the HERION code [20] uses the dipole and impulse approximations with relativistic Dirac-Fock wave functions. However, both codes neglect the magnetic interactions between colliding particles.

It is the aim of the present work to provide a theoretical framework for ionization in relativistic ion-atom collisions with possible account for the magnetic interactions for arbitrary many-electron ions. The formulas obtained can be used for ionization of projectile ions such as $\mathrm{U}^{28+}$ colliding with the rest-gas atoms and molecules at energies up to tens of $\mathrm{GeV} / \mathrm{u}$. Such heavy many-electron ions are of practical implications for the international FAIR project at GSI Darmstadt [21].

\section{FIRST-ORDER PERTURBATION THEORY OF RELATIVISTIC IONIZATION}

In the momentum-transfer $q$ representation, an accurate procedure to include the relativistic effects in ionization cross sections using the PWBA leads to the following result [5]:

$$
\begin{aligned}
\sigma(v)= & \frac{8 \pi a_{0}^{2} N_{n l}}{v^{2}} \int_{q_{0}}^{\infty} Z_{T}^{2}(q)\left[|F(q)|^{2}\right. \\
& \left.+\frac{\beta^{2}\left(1-q_{0}^{2} / q^{2}\right)}{\left(1-\beta^{2} q_{0}^{2} / q^{2}\right)^{2}}|G(q)|^{2}\right] \frac{d q}{q^{3}}, \\
v & =\beta c, \quad q_{0}=\left(I_{n l}+\epsilon\right) / v,
\end{aligned}
$$

where $a$ denotes the Bohr radius, $v$ the ion velocity, $c$ the speed of light, $\beta$ the relativistic factor, $n$ and $l$ the principal and orbital quantum numbers of the projectile electron shell with ionization potential $I_{n l}$ and number of equivalent electrons $N_{n l}$, respectively, $Z_{T}(q)$ the effective charge of the target which, in general, depends on $q$, and $q_{0}$ the minimal momentum transfer.

The matrix elements $F$ and $G$ are evaluated in Cartesian coordinate system where the $z$ axis is directed along the momentum transfer vector $\vec{q}$. The $x$ axis lies in the plane formed by the vectors $\vec{q}$ and $\vec{v}$. The matrix elements $F$ and $G$ are given by [5]

$$
\begin{gathered}
F(q)=\left\langle f\left|e^{i q z}\right| i\right\rangle, \\
G(q)=\left\langle f\left|\alpha_{x} e^{i q z}\right| i\right\rangle,
\end{gathered}
$$

where $|i\rangle$ and $|f\rangle$ denote the wave functions of the initial and final states of the projectile electron, and $\alpha_{x}$ the $x$ component of the Dirac matrix vector $\vec{\alpha}$.

The term proportional to $|F|^{2}$ is the main term used for nonrelativistic collisions, and the one proportional to $|G|^{2}$ is the relativistic modification describing the magnetic interactions between colliding particles. The matrix element $F$ is relatively easy to calculate and it is commonly used for cross-section calculations in PWBA, e.g., in the nonrelativistic Loss code [19] (Appendix, Sec. 1). In the LOSS-R code, ionization cross sections are calculated with the term $G=0$ 
but with relativistic effects accounted ad hoc by taking the minimal momentum transfer $q_{0}$ depending on the relativistic factor $\gamma$ (see [18] and Appendix, Sec. 2):

$$
q_{0}=\omega / \gamma v \text {. }
$$

The expression similar to Eq. (5) was obtained by Bethe [22] in analyzing the energy transferred to an atom in collision with a heavy particle.

In the LOSS-R code, the Schrödinger nonrelativistic radial wave functions are used for the projectile electrons in the bound and continuum states. This is justified for manyelectron heavy ions when the main contribution comes from ionization of outer-shell electrons (see next section).

The PWBA was also used in the calculation of bound-free pair production in antiproton-nucleus collisions (antihydrogen production) [23] and in heavy ion collisions [24]. Pair production may be considered as ionization of the negative energy Dirac "sea," and one has to use Dirac wave functions, rather than the Schrödinger wave functions.

Another treatment and wave functions are used in the HERION code [20] which applies the relativistic Dirac-Fock functions and where the relativistic ionization cross sections are calculated as a sum of dipole and nondipole parts in the impact-parameter representation:

$$
\sigma(v)=\sigma_{\text {dip }}(v)+\sigma_{\text {nondip }}(v) .
$$

The dipole part is expressed in terms of a photoionization cross $\sigma(\omega)$ and the equivalent-photon number $n(\omega)$ whereas the nondipole part is calculated in the impulse approximation (Appendix, Sec. 3).

In heavy-particle ionization, the main difficulty arises in calculation of the matrix element $G(q)$; this term was calculated only for ionization of projectile $1 s$ electron using various approximations (see, e.g., [4,10]). In the present work, the matrix element $G$ is evaluated for relativistic ionization of electron with arbitrary quantum numbers $n$ and $l$ in the nonrelativistic limit of $\alpha$-matrix element.

\section{NUMERICAL CALCULATIONS BY LOSS-R AND HERION CODES}

Relativistic ionization cross sections of $1 \mathrm{~s}$ electrons in $\mathrm{H}$-like $\mathrm{Pb}^{81+}$ ion by neutral atoms from $\mathrm{Be}$ to $\mathrm{Au}$ at $E$ $=158 \mathrm{GeV} / \mathrm{u} \quad(\gamma=170)$ as a function of the target nuclear charge $Z_{n}$ are shown in Fig. 1. Experimental data obtained at CERN are presented by open [11] and solid [12] circles. PWBA calculations [9] are shown by the dotted curve, relativistic model cross sections [15] by the dashed curve, and calculations with the Darwin wave functions [13] by stars. Curves 1 and 2 show results obtained by the LOSS-R and HERION codes, respectively. Experimental data agree with all theoretical results within 20\%-30\%.

Calculated partial (on projectile electron subshells $n l$ ) and total ionization cross sections of $\mathrm{U}^{28+}$ ions by protons at energies $E=1-100 \mathrm{GeV} / \mathrm{u}$ are shown in Fig. 2. Calculations were performed by the LOSS-R code using Eqs. (A6)-(A10). As seen from the figure, the main contribution to the total cross section is given by electron ionization from inner $3 d^{10}$, $4 p^{6}, 4 d^{10}$, and $4 f^{14}$ shells of $\mathrm{U}^{28+}$ which can be described by

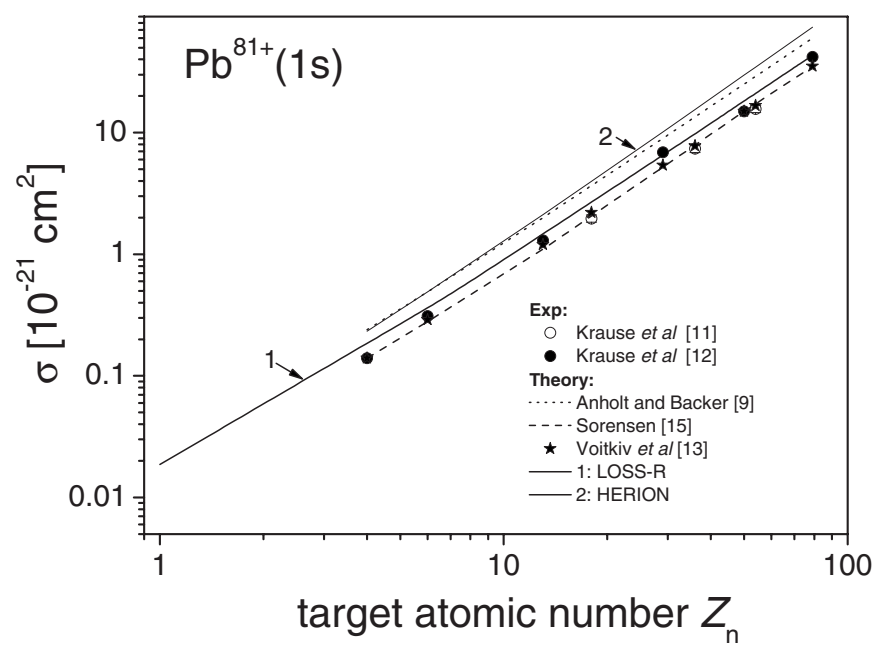

FIG. 1. Ionization cross sections of H-like $\mathrm{Pb}^{81+}(1 s)$ ions by neutral atoms with atomic numbers $4 \leq Z_{n} \leq 79$ at $E=158 \mathrm{GeV} / \mathrm{u}$ $(\gamma=170)$ as a function of the target atomic number $Z_{n}$. Experiment: open circles-[11], solid circles-[12]. Theory: dotted curveplane-wave Born approximation [9]; dashed curve-relativistic model [15]; stars - calculation with the Darwin relativistic wave functions [13]; curve 1-the Loss-R code, Eqs. (A1) and (A2); Eq. (A4), curve 2-the HERION code, Eqs. (A6)-(A10); Eq. (A8) with $Z_{T}^{2}=Z_{n}^{2}+Z_{n}$.

the nonrelativistic wave functions. In the energy range considered, relativistic ionization cross sections logarithmically increase with the ion energy increasing.

A comparison of relativistic ionization cross sections of $\mathrm{U}^{28+}$ ions by proton impact calculated by two different codes, LOSS-R and HERION, is displayed in Fig. 3 where the contributions from dipole and nondipole parts are shown together with the total (dipole+nondipole) cross sections. In the LOSS-R code, the dipole and nondipole parts of the ionization cross section correspond to sums over $\lambda$ in Eq. (24)

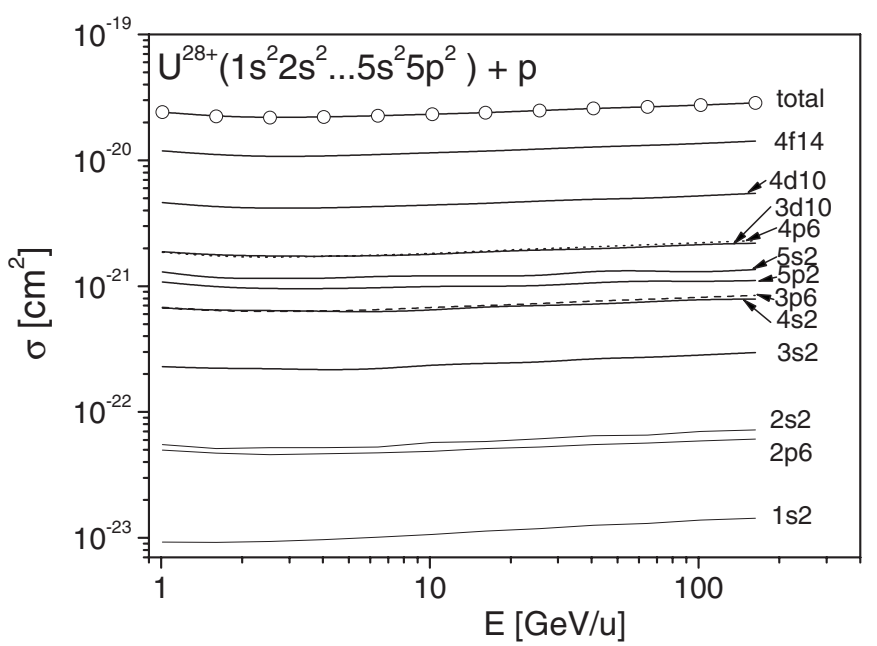

FIG. 2. Calculated ionization cross sections of $\mathrm{U}^{28+}\left(1 s^{2} \ldots 4 f^{14} 5 s^{2} 5 p^{2}\right)$ ions by proton impact as a function of ion energy: the LOSS-R-code [18], Eqs. (A6)-(A10). Contributions of ionization from different $n l$ shells of $\mathrm{U}^{28+}$ are shown together with the total cross section. 


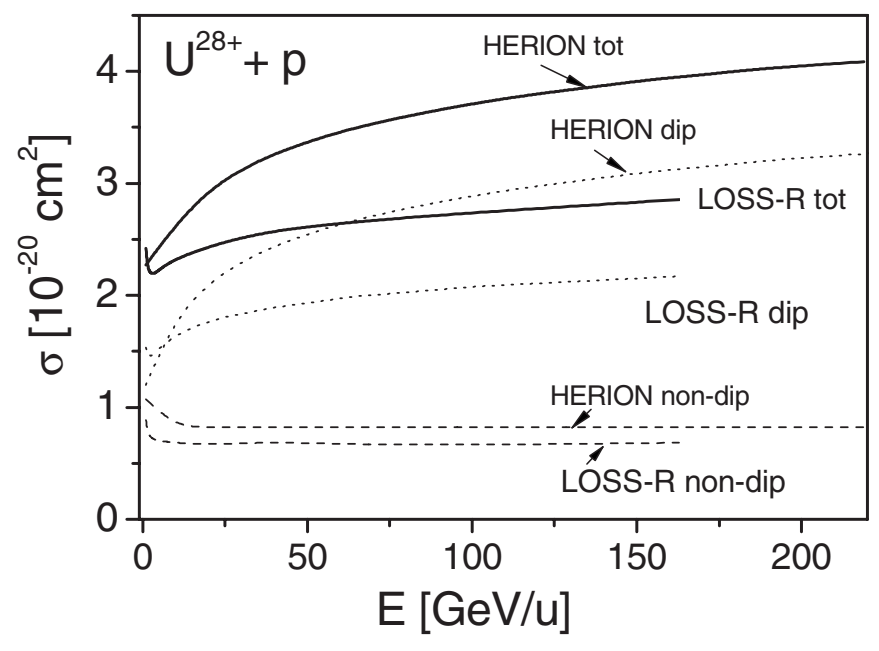

FIG. 3. Relativistic dipole, nondipole, and total ionization cross sections, i.e., summed over all projectile $n l$ shells, of $\mathrm{U}^{28+}$ by proton impact calculated by the HERION and LOSS-R codes (indicated) as a function of ion energy: dashed curves-the nondipole parts, dotted curves - the dipole parts, and solid curves - the total cross sections (see text).

with $\lambda=l \pm 1$ and $\lambda \neq l \pm 1$, respectively, where $\lambda$ is the orbital momentum of ejected electron.

As seen from Fig. 3, the nondipole parts calculated by the codes agree within $30 \%$ whereas the dipole parts and the total cross sections agree within a factor of 2. Most probably, this discrepancy is related to two reasons: first, the use of different wave functions in the codes, i.e., relativistic Dirac-Fock functions in HERION code and nonrelativistic Schrödinger functions in the LOSS-R code. Second, in the HERION code the dipole parts of ionization cross sections are calculated through the photoionization cross sections which are much higher than those calculated in the usual nonrelativistic approximation. This is due to the presence of the so-called giant resonances occurring as a result of correlation effects between different electronic subshells. The effects are quite large, especially in heavy many-electron atomic systems (see [25] for details). We note that the dipole part of the relativistic ionization cross sections involves about $60 \%-70 \%$ of the total cross section while the nondipole part has a weak dependence on energy and, therefore, the total cross section has practically the same shape as the dipole part.

Experimental, theoretical relativistic and nonrelativistic ionization cross sections of $\mathrm{H}-$ like $\mathrm{Au}^{78+}(1 s)$ ions colliding with carbon atoms are displayed in Fig. 4. The nonrelativistic result (the LOSS code) shows a Born maximum followed by a decrease $\left(\sim \ln v / v^{2}\right)$ of the cross section. The relativistic result obtained with the LOSS-R code has a local minimum around $1 \mathrm{GeV} / \mathrm{u}$ and increases logarithmically with energy. The results obtained with the HERION code have a little better agreement with experiment at $E>200 \mathrm{MeV} / \mathrm{u}$ than those by the LOSS-R code. In the calculations by the HERION code, the effective charge of the carbon target $Z_{T}^{2}=Z_{n}^{2}+Z_{n}=42$ was used whereas in the LOSS-R code the target effective charge $Z_{T}(q)$ is calculated as a function of the momentum transfer $q$ [see Eq. (A2)], i.e., with account for screening and antis-

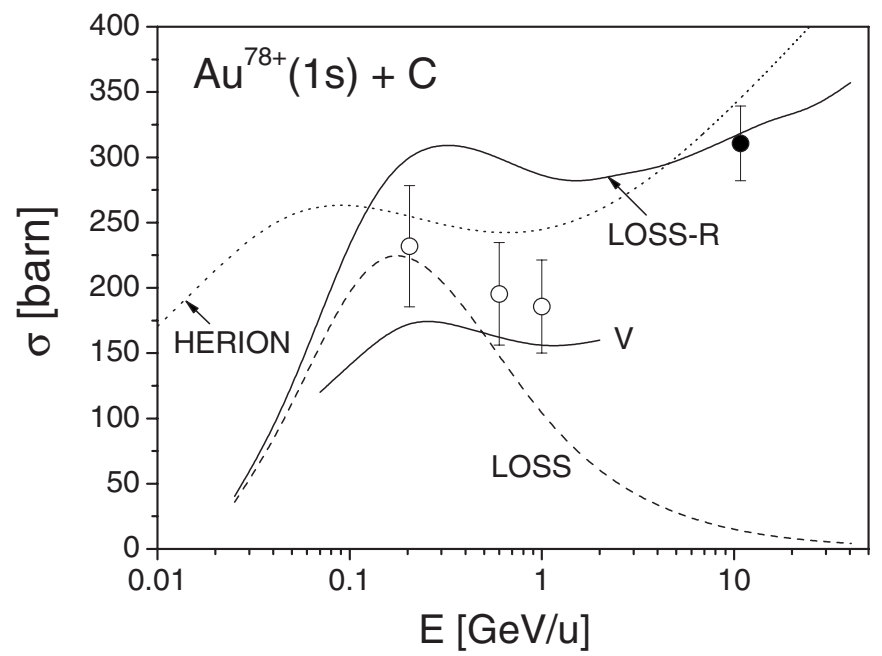

FIG. 4. Ionization cross sections of H-like $\mathrm{Au}^{78+}(1 s)$ ions by carbon atoms as a function of ion energy. Experiment: open circles-[14], [26]; solid circle- [27]. Theory: Lossnonrelativistic LOSS code, Eqs. (A1) and (A2); LOSS-R-relativistic LOSS-R code, Eqs. (A1), (A2), and (A4); HERION-HERION code, Eqs. (A6)-(A10); Eq. (A8) with the carbon effective charge $Z_{T}^{2}$ $=42$; curve $\mathrm{V}$-calculations by Voitkiv [17] with relativistic electron description and $Z_{T}^{2}=36$.

creening effects; here $Z_{n}$ denotes the target atomic number.

The curve V in Fig. 4 is the recent result [17] obtained on the basis of relativistic electron description and Dirac wave functions with $Z_{T}=6$. As is seen, the agreement of results obtained by both the HERION code and in the work [17] with experimental data is nearly the same, i.e., within a factor of 1.5. We note that calculations [17] are presented only up to energy of $2 \mathrm{MeV} / \mathrm{u}$ that makes it difficult to make a comparison with experimental point at $E=10 \mathrm{MeV} / \mathrm{u}$ and present calculations.

\section{NONRELATIVISTIC LIMIT OF $\alpha$-MATRIX ELEMENT}

In this section, the relativistic matrix element $G(q)$, Eq. (4), will be evaluated for ionization of projectile electron with arbitrary quantum numbers $n$ and $l$ in the nonrelativistic limit of $\alpha$-matrix element. The operator $J=\alpha_{x} \exp (i q z)$ in Eq. (4) is odd and connects the large and small components of the Dirac wave function. It turns to zero if one uses only the large component. The nonrelativistic limit of $J$ is given in the form [28]

$$
\begin{gathered}
J=\frac{1}{2 m}\left[\alpha_{x} e^{i q z} \rho_{1}(\vec{\sigma} \cdot \vec{p})+(\vec{\sigma} \cdot \vec{p}) \rho_{1} \alpha_{x} e^{i q z}\right], \\
\vec{\sigma}=\left(\sigma_{x}, \sigma_{y}, \sigma_{z}\right) \equiv\left(-i \alpha_{y} \alpha_{z},-i \alpha_{z} \alpha_{x},-i \alpha_{x} \alpha_{y}\right),
\end{gathered}
$$

where $\rho_{1}=\sigma_{z} \alpha_{z}$. The operator $\vec{p}=\vec{e}_{z} q$ acts only on the large component of the Dirac wave function. Using relations $\alpha_{x} \rho_{1}=\sigma_{x}$ and $\rho_{1} \alpha_{x}=\sigma_{x}$, one obtains

$$
J=\frac{1}{2 m c}\left[\sigma_{x} e^{i q z}(\vec{\sigma} \cdot \vec{p})+(\vec{\sigma} \cdot \vec{p}) \sigma_{x} e^{i q z}\right] .
$$

Using the identity 


$$
(\vec{\sigma} \cdot \vec{A})(\vec{\sigma} \cdot \vec{B})=(\vec{A} \cdot \vec{B})+i \vec{\sigma} \cdot(\vec{A} \times \vec{B})
$$

with $\vec{A}=\vec{e}_{x}, \vec{B}=\vec{p}$ and $\vec{B}=\vec{e}_{x}, \vec{A}=\vec{p}$, we find

$$
J=\frac{1}{2 m}\left[e^{i q z} p_{x}+p_{x} e^{i q z}+(\text { spin-flip terms })\right]
$$

where the spin-flip terms are given by

$$
e^{i q z}(\vec{p} \times \vec{\sigma})_{x}-(\vec{p} \times \vec{\sigma})_{x} e^{i q z} .
$$

The spin-flip terms appear in the Pauli approximation from the Dirac equation. In the following, we neglect these terms because they are small in our case (see also [4]).

Taking into account that $p_{x}=-i \hbar \frac{\partial}{\partial x}$ and $\frac{\partial}{\partial x} e^{i q z}=0$, one has for the nonrelativistic limit of $J$ operator:

$$
J=\frac{1}{2 m}\left(e^{i q z} p_{x}+p_{x} e^{i q z}\right)
$$

This result can be also obtained directly using the electromagnetic-current approach in the Schrödinger theory (see, e.g., [29]).

After these assumptions, the matrix element $G(q)$ becomes

$$
G(q)=\frac{-i \hbar}{m c}\left\langle f\left|e^{i q z} \frac{\partial}{\partial x}\right| i\right\rangle .
$$

We note that it is easier to calculate the derivative $\frac{\partial}{\partial z}$ in spherical coordinates rather than $\frac{\partial}{\partial x}$, so we rotate the coordinate system by $\pi / 2$ around the $y$ axis. This changes $x \rightarrow z$ and $z \rightarrow-x$, and we have

$$
G(q)=\frac{-i \hbar}{m c}\left\langle f\left|e^{-i q x} \frac{\partial}{\partial z}\right| i\right\rangle .
$$

Actually, we need to obtain the $|G(q)|^{2}$ value summed over spins and integrated over the angle of the outgoing electron. This matrix element is calculated here according to the general rules of angular-momentum algebra (see, e.g., [30]).

Let us present the bound $|i\rangle$ and continuous $|f\rangle$ wave functions in the form of separated angular and radial parts which correspond to the central-field approximation,

$$
|i\rangle=Y_{l m}(\hat{\mathbf{r}}) P_{n l}(r) / r, \quad|f\rangle=Y_{\lambda \mu}(\hat{\mathbf{r}}) P_{\epsilon \lambda}(r) / r,
$$

with normalizations

$$
\int P_{n l}^{2}(r) d r=1, \quad \int P_{\epsilon \lambda}(r) P_{\epsilon^{\prime} \lambda}(r) d r=\pi \delta\left(\epsilon-\epsilon^{\prime}\right) .
$$

Here $Y_{a b}(\hat{\mathbf{r}})$ denotes a spherical harmonic, $P(r)$ the radial wave function, and $\lambda$ the angular momentum of ejected electron.

According to [30], the action of the derivative $\partial / \partial z$ on the bound wave function $|i\rangle$ yields

$$
\begin{aligned}
\partial|i\rangle / \partial z= & \left(\frac{l+1}{\sqrt{(2 l+1)(2 l+3)}} Y_{l+1 m}\right. \\
& \left.+\frac{l}{\sqrt{(2 l-1)(2 l+1)}} Y_{l-1 m}\right) d P_{n l}(r) / d r \\
& -\left(\frac{l(l+1)}{\sqrt{(2 l+1)(2 l+3)}} Y_{l+1 m}\right. \\
& \left.-\frac{l(l-1)}{\sqrt{(2 l-1)(2 l+1)}} Y_{l-1 m}\right) P_{n l}(r) / r .
\end{aligned}
$$

We note that the present procedure is similar to that used for calculating the magnetic terms of the pionium breakup [31].

Expanding exponent in Eq. (15) over spherical harmonics $Y_{\kappa \mu}$ and making integration over angles give

$$
\begin{aligned}
G(q)= & \sum_{\kappa \mu \lambda m_{\lambda}} \frac{4 \pi^{2}}{q} i^{\kappa} Y_{\kappa \mu}^{*}(\hat{x}) Y_{\lambda m_{\lambda}}^{*}(\hat{q})\left\{\frac { l + 1 } { \sqrt { ( 2 l + 1 ) ( 2 l + 3 ) } } \Omega _ { + } \left(R^{d}\right.\right. \\
& \left.\left.-l R^{B}\right)+\frac{l}{\sqrt{(2 l-1)(2 l+1)}} \Omega_{-}\left[R^{d}-(l-1) R^{B}\right]\right\}, \quad(19) \\
\Omega_{ \pm} & =\sqrt{\frac{(2 l+1)(2 \lambda+1)(2 \kappa \pm 1)}{4 \pi}}\left(\begin{array}{ccc}
\lambda & \kappa & l \pm 1 \\
m_{\lambda} & \mu & m
\end{array}\right) \\
& \times\left(\begin{array}{ccc}
\lambda & \kappa & l \pm 1 \\
0 & 0 & 0
\end{array}\right) .
\end{aligned}
$$

Here $R^{B}$ and $R^{d}$ denote two types of radial matrix elements: a "usual" (Born) one

$$
R^{B}(q)=\int_{0}^{\infty} P_{\epsilon \lambda}(r)\left[j_{\kappa}(q r)-\delta_{\kappa 0}\right] P_{n l}(r) d r,
$$

and a "new" one involving the derivative of the initial radial wave function:

$$
R^{d}=\int_{0}^{\infty} P_{\epsilon \lambda}(r) j_{\kappa}(q r) \frac{d P_{n l}(r)}{d r} d r .
$$

Using the sum rules for $3 j$ symbols and making summation over projections $m$ and $m_{\lambda}$, one obtains for $|G(q)|^{2}$ :

$$
\begin{aligned}
|G(q)|^{2}= & \sum_{\kappa \lambda} \frac{2}{c^{2}} \frac{(2 \kappa+1)(2 \lambda+1)}{2 l+1} \mid i^{\kappa}\left(\begin{array}{ccc}
\lambda & \kappa & l+1 \\
0 & 0 & 0
\end{array}\right)[(l \\
& \left.+1) R^{d}(q)+l(l-1) R^{B}(q)\right]+i^{\kappa}\left(\begin{array}{ccc}
\lambda & \kappa & l-1 \\
0 & 0 & 0
\end{array}\right) \\
& \times\left.\left[l R^{d}(q)+l(l-1) R^{B}(q)\right]\right|^{2}
\end{aligned}
$$

Finally, the relativistic ionization cross section from the arbitrary projectile electronic shell $n l$ with $N_{n l}$ equivalent electrons by a heavy target particle with an affective charge $Z_{T}(q)$ including magnetic interactions can be written in form (1) where the relativistic part $|G(q)|^{2}$ is described by Eq. (23) and the nonrelativistic part $|F(q)|^{2}$ is given by 


$$
\begin{gathered}
|F(q)|^{2}=\sum_{\kappa \lambda}(2 \kappa+1)(2 \lambda+1)\left(\begin{array}{ccc}
l & \lambda & \kappa \\
0 & 0 & 0
\end{array}\right)^{2}\left|R^{B}(q)\right|^{2}, \\
|l-\lambda| \leq \kappa \leq l+\lambda .
\end{gathered}
$$

Equations (1), (23), and (24) will be realized in the new computer code preliminary entitled RICODE: relativistic ionization code.

\section{CONCLUSION}

Relativistic ionization of heavy highly charged ions colliding with atoms and ions is considered. Numerical calculations performed by newly developed LOSS-R and HERION codes for relativistic ionization of $1 s$ electron in $\mathrm{H}$-like ions show a quite good agreement with experimental data. Using these codes, calculations were made for relativistic ionization of projectile electrons with the quantum numbers $n l$ $=1 s, 2 s, \ldots, 5 s$, and $5 p$ for $\mathrm{U}^{28+}+p$ collisions showing an overall agreement between codes within a factor of 2 .

Formulas were derived for the relativistic ionization cross sections for an arbitrary $n l$ shell of the projectile ion with account for the magnetic interactions between projectile ion and the target atom or ion using the nonrelativistic limit of the Dirac matrix element. The ionization cross section is presented in the momentum-transfer $q$ representation using two terms: the usual Born approximation [matrix element $F(q)$ ] and the relativistic term $G(q)$ responsible for the magnetic interactions. The $G(q)$ term is expressed through the $x$ component of the Dirac matrix vector $\vec{\alpha}$. Both terms are presented in the form of separated angular and radial parts and the $G(q)$ term is expressed via integral of the continuumstate wave function and the derivative of the initial bound wave function. The derived formulas will be realized in the computer code which is under development now.

\section{ACKNOWLEDGMENTS}

The authors are grateful to S. N. Andreev for the help in the codes development. This work was performed under INTAS Grant No. 06-1000012-8530 and RFBR Grant No. 0802-00005-a.

\section{APPENDIX: COMPUTER CODES LOSS, LOSS-R AND HERION}

\section{LOsS code}

The LOSS code [19] is intended for calculation of singleelectron ionization of projectile ions in collisions with atoms and ions at nonrelativistic energies $E<100 \mathrm{MeV} / \mathrm{u}$ using the plane-wave Born approximation in the momentumtransfer $q$ representation,

$$
\sigma(v)=\frac{8 \pi a_{0}^{2} N_{n l}}{v^{2}} \int_{0}^{\infty} d \epsilon \int_{q_{0}}^{\infty} \frac{d q}{q^{3}}|F(q)|^{2}\left|Z_{T}(q)\right|^{2},
$$

where $F(q)$ is defined in Eqs. (21) and (24). Summation over multipoles $\kappa$ in Eq. (24) is made up to $\kappa_{\max }=4$, and over the orbital momenta of ejected electron up to $\lambda_{\max }=12$.
The effective target charge $Z_{T}(q)$ and minimum momentum transfer $q_{0}$ are given by

$$
\begin{aligned}
\left|Z_{T}(q)\right|^{2}= & {\left[Z_{n}-\sum_{j=1}^{N}\left\langle j\left|e^{i \mathbf{q r}}\right| j\right\rangle\right]^{2}+\left[N-\sum_{j=1}^{N}\left\langle j\left|e^{(i \mathbf{q r}}\right| j\right\rangle\right] } \\
& q_{0}=\left(I_{n l}+\epsilon\right) / v
\end{aligned}
$$

Here $Z_{n}$ and $N$ denote the target atomic number and the number of electrons, respectively. For neutral atoms $Z_{n}=N$ and for protons $Z_{n}=1, N=0$.

In the LOSS code, the wave functions of the bound $|n l\rangle$ and continuum $|\epsilon \lambda\rangle$ states of the projectile are found by numerical solution of the nonrelativistic Schrödinger equation, and those for the target $|j\rangle$ are calculated using the nodeless Slater wave functions. The atomic structure of the target is taken into account through the effective charge $Z_{T}(q)$, Eq. (A2).

In the LOSS code, ionization cross sections decrease with the projectile energy increasing in accordance with the Born approximation:

$$
\sigma(v) \rightarrow \frac{\ln v}{v^{2}}, \quad E \rightarrow \infty, \quad v \rightarrow \infty
$$

\section{LOSS-R code}

The LOSS-R code (relativistic loss) is the relativistic version of the LOSS code (see Appendix, Sec. 1) which uses the same Eqs. (A1), (24), and (A2) but with the following changes (see [18]):

$$
v=\beta c, \quad q_{0}=\frac{I_{n l}+\epsilon}{\gamma v}, \quad \gamma=\frac{1}{\sqrt{1-\beta^{2}}}=1+\frac{E}{m_{0} c^{2}},
$$

where $\beta$ and $\gamma$ are relativistic factors, $E$ denotes the projectile kinetic energy per nucleon, $m_{0}$ the atomic mass unit, $m_{0} c^{2}=931.494 \mathrm{MeV}$. In the LOSS-R code the relativistic effects are accounted through two factors: relativistic velocity $v$, which is constant at $E \rightarrow \infty$, and dependence of the minimum momentum transfer $q_{0}$ on $\gamma$ according to Eq. (A4).

At relativistic energies, the LOSS-R code provides the following asymptotic behavior:

$$
\sigma(v) \rightarrow \ln \gamma, \quad E \rightarrow \infty, \quad v \rightarrow c .
$$

In the LOSS-R code, the nonrelativistic Schrödinger wave functions are used and the magnetic interactions are not accounted for. At nonrelativistic energies the results given by the LOSS-R code coincide with those obtained by the LOSS code.

\section{HERION code}

The HERION code is intended for calculation of relativistic cross sections of ionization of projectile ions in collisions with atoms and ions at relativistic energies $E>100 \mathrm{MeV} / \mathrm{u}$ using the relativistic Dirac-Fock wave functions (see [20]).

In the HERION code, the relativistic ionization cross sections are calculated as a sum of dipole and nondipole parts, 


$$
\sigma(v)=\sigma_{\text {dip }}(v)+\sigma_{\text {nondip }}(v) .
$$

The dipole part is expressed in terms of a photoionization cross $\sigma(\omega)$ and the equivalent-photon number $n(\omega)$ (see [6]):

$$
\begin{gathered}
\sigma_{d i p}(v)=\int_{\omega_{\min }}^{\infty} n(\omega) \sigma(\omega) \frac{d \omega}{\omega}, \\
n(\omega)=\frac{2 Z_{T}^{2}}{\pi(\beta c)^{2}}\left\{x K_{0}(x) K_{1}(x)-\frac{1}{2}(\beta x)^{2}\left[K_{1}^{2}(x)-K_{0}^{2}(x)\right]\right\}, \\
x=\frac{\omega_{\min } b_{\min }}{\gamma \beta c} .
\end{gathered}
$$

As was mentioned in Sec. III, photoionization cross sections in HERION code are calculated with account for correlation effects between different electronic subshells. The minimum impact parameter $b_{\min }$ is defined by the mean size of the $n l$ shell and is given with a good accuracy by

$$
b_{\min } \approx \frac{n}{\sqrt{2 I_{n l}}} a_{0} .
$$

The nondipole part is calculated in the impulse approximation (see [20]):

$$
\sigma_{\text {nondip }}(v)=2 \pi a_{0}^{2} \frac{N_{n l}}{I_{n l}}\left(\frac{Z_{T} \alpha}{\beta}\right)^{2} .
$$

The target effective charge is considered a constant value equal to $Z_{T}^{2}=Z_{n}^{2}+N$, i.e., independent of the momentum transfer $q$. In the HERION code, relativistic ionization cross sections are calculated without account for the magnetic interactions.
[1] Atomic Physics with Heavy Ions, edited by H. Beyer and V. P. Shevelko (Springer, Berlin, 1999).

[2] The Physics of Multiply and Highly Charged Ions, edited by F. J. Currell (Kluwer Academic, Dordrecht, 2003).

[3] G. Kraft, Prog. Part. Nucl. Phys. 45, S473 (2000).

[4] R. Anholt, Phys. Rev. A 19, 1004 (1979).

[5] J. Eichler and W. E. Meyerhof, Relativistic Atomic Collisions (Academic Press, New York, 1995).

[6] C. A. Bertulani and G. Baur, Phys. Rep. 163, 299 (1988).

[7] A. B. Voitkiv, Phys. Rep. 392, 191 (2004).

[8] R. Anholt, W. E. Meyerhof, H. Gould, C. Munger, J. Alonso, P. Thieberger, and H. E. Wegner, Phys. Rev. A 32, 3302 (1985).

[9] R. Anholt and U. Becker, Phys. Rev. A 36, 4628 (1987).

[10] D. M. Davidovic, B. L. Moiseiwitsch, and P. H. Norrington, J. Phys. B 11, 847 (1978).

[11] H. F. Krause, C. R. Vane, S. Datz, P. Grafstrom, H. Knudsen, C. Scheidenberger, and R. H. Schuch, Phys. Rev. Lett. 80, 1190 (1998).

[12] H. F. Krause, C. R. Vane, S. Datz, P. Grafstrom, H. Knudsen, U. Mikkelsen, C. Scheidenberger, R. H. Schuch, and Z. Vilakazi, Phys. Rev. A 63, 032711 (2001).

[13] A. B. Voitkiv, C. Müller, and N. Grün, Phys. Rev. A 62 062701 (2000).

[14] Th. Stöhlker et al., Nucl. Instrum. Methods Phys. Res. B 124, 160 (1997).

[15] A. H. Sorensen, Phys. Rev. A 58, 2895 (1998).

[16] S. Fritzsche, A. Surzhykov, and Th. Stöhlker, Nucl. Instrum. Methods Phys. Res. B 205, 469 (2003).
[17] A. B. Voitkiv, J. Phys. B 40, 2885 (2007).

[18] I. L. Beigman, I. Yu. Tolstikhina, and V. P. Shevelko, J. Tech. Phys. 53, 547 (2008).

[19] V. P. Shevelko, I. Yu. Tolstikhina, and Th. Stöhlker, Nucl. Instrum. Methods Phys. Res. B 184, 295 (2001).

[20] S. N. Andreev, I. L. Beigman, I. Yu. Tolstikhina, V. P. Shevelko, G. Baur, and Th. Stöhlker, Bull. Lebedev Phys. Inst. 35, 89 (2008).

[21] http://www.gsi.de/fair/index_e.html

[22] H. Bethe, Handbuch der Physik (Springer, Berlin, 1933), Vol. 24, p. 273.

[23] H. Meier et al., Eur. Phys. J. C 5, 287 (1998).

[24] H. Meier, Z. Halabuka, K. Hencken, D. Trautmann, and G. Baur, Phys. Rev. A 63, 032713 (2001).

[25] M. Amusya, Atomic Photoeffect (Plenum, New York, 1990).

[26] C. Scheidenberger et al., Nucl. Instrum. Methods Phys. Res. B 90, 36 (1994).

[27] N. Claytor, A. Belkacem, T. Dinneen, B. Feinberg, and H. Gould, Phys. Rev. A 55, R842 (1997).

[28] A. Messiah, Quantum Mechanics (North-Holland, Amsterdam, 1962), Vol. 2.

[29] V. B. Berestetskii, E. M. Lifshitz, and L. P. Pitaevskii, Relativistic Quantum Theory (Pergamon, Oxford, 1974).

[30] A. R. Edmonds, Angular Momentum in Quantum Mechanics (Princeton University Press, Princeton, 1996).

[31] T. A. Heim, K. Hencken, D. Trautmann, and G. Baur, J. Phys. B 34, 3763 (2001). 